ACT- $11 / 98$

CTP-TAMU-45/98

TPI-MINN-98/24

UMN-TH-1729-98

hep-ph/9811427

November 1998

\title{
String Derived MSSM and M-theory Unification
}

\author{
G.B. Cleaver, ${ }^{1,2}$ A.E. Faraggi, $3 \mathrm{~T}^{\circ}$ and D.V. Nanopoulos ${ }^{1,2,4}$ \\ ${ }^{1}$ Center for Theoretical Physics, Dept. of Physics, Texas A\&M University, \\ College Station, TX 77843, USA \\ ${ }^{2}$ Astro Particle Physics Group, Houston Advanced Research Center (HARC), \\ The Mitchell Campus, Woodlands, TX 77381, USA \\ ${ }^{3}$ Department of Physics, University of Minnesota, Minneapolis, MN 55455, USA \\ ${ }^{4}$ Academy of Athens, Chair of Theoretical Physics, Division of Natural Sciences, \\ 28 Panepistimiou Avenue, Athens 10679, Greece
}

\begin{abstract}
The recent conjecture of possible equivalence between the string scale $M_{S}$ and the minimal supersymmetric standard model unification scale $M_{U} \approx$ $2.5 \times 10^{16} \mathrm{GeV}$ is considered in the context of string models. This conjecture suggests that the observable gauge group just below the string scale should be $S U(3)_{C} \times S U(2)_{L} \times U(1)_{Y}$ and that the $S U(3)_{C} \times S U(2)_{L} \times U(1)_{Y}$-charged spectrum of the observable sector should consist solely of the MSSM spectrum. We demonstrate that string models can actually be constructed that possess these observable features. Two aspects generic to many classes of three family $S U(3)_{C} \times S U(2)_{L} \times U(1)_{Y}$ string models are both an extra local anomalous $U(1)_{A}$ and numerous (often fractionally charged) exotic particles beyond the MSSM. Thus, for these classes, the key to obtaining an $M_{S}=M_{U} \approx 2.5 \times 10^{16}$ $\mathrm{GeV}$ string model is the existence of $F$ - and $D$-flat directions that near the string scale can simultaneously break the anomalous $U(1)$ and give mass to all exotic SM-charged observable particles, decoupling them from the low energy spectrum. In this letter we show, in the context of free fermionic strings, that string models with flat directions possessing these features do exist. We present one such string derived model in which all such exotic observable states beyond the MSSM receive mass at the scale generated by the Fayet-Iliopoulos term. The associated $F$ - and $D$-flat direction is proven flat to all orders of the superpotential.
\end{abstract}

To appear in Physics Letters B.

*gcleaver@rainbow.physics.tamu.edu

${ }^{\dagger}$ faraggi@mnhepw.hep.umn.edu

${ }^{\ddagger}$ dimitri@soda.physics.tamu.edu 


\section{Introduction}

One of the intriguing hints for new physics beyond the Standard Model (SM) is the nearly perfect unification of the Standard Model gauge couplings, assuming the spectrum of the Minimal Supersymmetric Standard Model (MSSM) above the electroweak scale. This coupling unification occurs at the scale of order $M_{\mathrm{MSSM}} \approx 2 \times 10^{16} \mathrm{GeV}$, and is one or two orders of magnitude below the scale where the gravitational interaction becomes comparable in strength to the gauge interactions. Gravity, on the other hand, can be unified consistently with the gauge interactions only if the MSSM is embedded in superstring/M-theory. However, a general prediction of perturbative string theory is that the gauge couplings unify at a scale $M_{S}$ of the order of $5 \times 10^{17} \mathrm{GeV}$. Thus, at least in the context of perturbative string theory it is natural to seek string models that contain the MSSM spectrum plus additionally a few color and electroweak states in vector-like representations. Such states can then receive intermediate mass scale, and elevate the coupling unification scale to the string scale while keeping the MSSM running coupling strengths in agreement with their experimentally measured $M_{Z^{\circ}}$ scale values. However, while string derived models that allow such scenarios have indeed been constructed, one may argue that the additional mass scales require additional ad hoc fine tuning, and therefore is not very attractive.

It is then quite remarkable that within the context of M-theory a mechanism has been proposed that allows the gauge couplings to unify at $\sim 10^{16} \mathrm{GeV}$, [1] thus maintaining the successful MSSM prediction. Alas, from the point of view of M-theory unification this would require that the observable gauge group after compactification is precisely $S U(3)_{C} \times S U(2)_{L} \times U(1)_{Y}$, which means that there should be no GUT or semi-GUT below the compactification (i.e., string) scale. Furthermore, it implies that soon below this scale the (SM-charged) spectrum in the observable sector must consist solely of the MSSM spectrum, i.e. three generations plus two electroweak Higgs doublets. However, string models generically give rise to several Higgs multiplets and to many exotic states, charged under the Standard Model, and which may remain massless at the string scale. In fact, to date it is in general claimed that there does not exist a single string model that produces solely the MSSM spectrum in the observable sector below the string scale.

In this letter we therefore undertake the task of deriving a string model that produces only the MSSM spectrum in the $S U(3)_{C} \times S U(2)_{L} \times U(1)_{Y}$-charged observable sector. The model that we study (referred to henceforth as the "FNY model") is the string model of [2] and was constructed in the free fermionic formulation. Like all other string models which are constructed by utilizing characters of level one KacMoody current algebra, it contains exotic fractionally charged states in the massless

*By observable sector we mean the part which affects the Standard Model gauge coupling evolution, thus allowing the existence of unbroken horizontal $U(1)$ symmetries, which do not mix with the Standard Model gauge group. Such symmetries do not survive to low energies in the model that we discuss, but it is a possibility that may occur a priori. 
spectrum. As pointed out in ref. [3], in this model all the exotic fractionally charged states couple to a set of Standard Model singlets at the cubic level of the superpotential. Thus, by assigning vacuum expectation values (VEVs) to this set of Standard Model singlets all the exotic fractionally charged states receive mass of the order of the string scale and decouple from the massless spectrum.

The model also contains, at the massless string level, a number of electroweak Higgs doublets and a color triplet/anti-triplet pair beyond the MSSM. We show that by the same suitable choice of flat directions that only one Higgs pair remains light below the string scale. The additional color triplet/anti-triplet pair receives mass from a fifth order superpotential term. This results in the triplet pair receiving a mass that is slightly below the string scale and is perhaps smaller than the doublet and fractional exotic masses by a factor of around $(1 / 10-1 / 100)$.

Thus, all $S U(3)_{C} \times S U(2)_{L} \times U(1)_{Y}$-charged non-MSSM states decouple from the massless spectrum at or slightly below the string scale by coupling to flat direction VEVs. Thus, for the first time we present a string model that yields below the string scale solely the spectrum of the MSSM in the observable sector. Such a string model can therefore serve as an example which satisfies the requirements imposed by the M-theory motivated unification.

Another interesting property of the FNY model is that provided that the weakhypercharge is left unbroken at the string scale, the additional $U(1)_{Z^{\prime}}$ which is embedded in $S O(10)$ is necessarily broken by non-Abelian singlet flat directions near the string scale. Thus, in this model the $S O(10)$ subgroup below the string scale is necessarily $S U(3) \times S U(2) \times U(1)_{Y}$, rather than $S U(3) \times S U(2) \times U(1)_{C} \times U(1)_{L}$, which is another appealing property from the point of view of M-theory motivated unification.

There is a crucial new input [4], which we now discuss, that allows us to achieve, at present, our goal of a string-derived MSSM, nearly a decade after the original model was constructed. Let us recall that this string model contains an anomalous $U(1)$ symmetry [5] (a generic feature of three family $S U(3)_{C} \times S U(2)_{L} \times U(1)_{Y}$ string models of free fermionic, bosonic lattice, or orbifold construction). Elimination of the anomalous $U(1)$ symmetry generates a Fayet-Iliopoulos (FI) term, by the VEV of the dilaton field, that breaks supersymmetry near the string scale. To preserve supersymmetry near the string scale, one must satisfy $F$ - and $D$-flatness constraints arising from the superpotential, by giving VEVs to a set of standard model scalar singlets in the massless spectrum of the string models [6]. Since these fields are typically also charged under the non-anomalous gauge symmetries, a non-trivial set of constraints is imposed on the possible choices of VEVs and in general will break some or all of these symmetries spontaneously. It turns out that in the FNY model of ref. [2] the D-flatness constraints are particularly restrictive in the following sense. In many free fermionic models utilizing the NAHE set of boundary condition basis vectors, one can solve the $D$-term constraints by assigning VEVs solely to $S O(10)$ singlet fields [7, 8]. However, it turns out, as we discuss below, that this is not possible 
in the model of ref. [2]. In the FNY model one must assign non-trivial VEVs to states which are Standard Model singlets, but that are charged under the $U(1)_{Z^{\prime}}$ which is embedded in $S O(10)$, thus complicating the problem of finding FNY flat directions. In fact, the original motivation to construct the model of ref. [8] was precisely to find flat $F$ and $D$ directions in the free fermionic standard-like models. However, recently the search for flat $F$ and $D$ directions in the free fermionic models has been systematized [4]. (See also [9, 10].) Thus revealing that the FNY model does admit flat $F$ and $D$ solutions, but that these solutions necessarily break the $U(1)_{Z^{\prime}}$ (as long as $U(1)_{Y}$ is preserved) which is embedded in $S O(10)$.

In this paper we therefore utilize the important new developments of the tools needed to find flat $F$ and $D$ solutions in the string models. To achieve our goal of deriving the MSSM directly from string theory, we have to incorporate the set of fields needed to give mass to the fractionally charged states into the possible solutions. Similarly we will impose that the generated VEVs also give large mass, of the order of the string scale, to the additional color triplets and electroweak doublets beyond the MSSM spectrum. We should comment that in other semi-realistic free fermionic models, the exotic states may also receive large mass, but often only through high order nonrenormalizable terms, and therefore their respective intermediate mass scales will be highly suppressed relative to the string scale. Therefore, in the present letter, for the first time, we are able to construct a string solution with solely the MSSM spectrum in the $S U(3) \times S U(2) \times U(1)_{Y}$-charged observable sector below the scale generated by the Fayet-Iliopoulos term, a scale on par with the string scale.

\section{The model}

\subsection{Model construction}

The FNY string model [2] was constructed in the free fermionic formulation [11]. It is generated by a set of eight basis vectors of boundary conditions for all the worldsheet free fermions. The first five vectors consist of the NAHE set $\left\{\mathbf{1}, \mathbf{S}, \mathbf{b}_{1}, \mathbf{b}_{2}, \mathbf{b}_{3}\right\}$ [12]. In addition the basis contains three additional vectors which are displayed in Table (2.1)

\begin{tabular}{|c|c|c|c|c|c|c|c|c|c|}
\hline & $\psi^{\mu}$ & $\chi^{12}$ & $\chi^{34}$ & $\chi^{56}$ & $\bar{\psi}^{1, \ldots, 5}$ & $\bar{\eta}^{1}$ & $\bar{\eta}^{2}$ & $\bar{\eta}^{3}$ & $\bar{\phi}^{1, \ldots, 8}$ \\
\hline $\mathbf{b}_{4}$ & 1 & $\overline{c 1}$ & 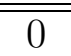 & $\overline{c 0}$ & $\begin{array}{lllll}11 & 1 & 1 & 1\end{array}$ & 1 & 0 & 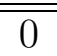 & $\begin{array}{lllllllll} & 0 & 0 & 0 & 0 & 0 & 0 & 0\end{array}$ \\
\hline$\beta$ & 1 & 0 & 0 & 1 & 11100 & 1 & 0 & 1 & 11110000 \\
\hline$\gamma$ & 1 & 0 & 1 & 0 & $\begin{array}{lllll}\frac{1}{2} & \frac{1}{2} & \frac{1}{2} & \frac{1}{2} & \frac{1}{2}\end{array}$ & $\frac{1}{2}$ & $\frac{1}{2}$ & $\frac{1}{2}$ & $\frac{1}{2} 0111 \frac{1}{2} \frac{1}{2} \frac{1}{2} 1$ \\
\hline
\end{tabular}




\begin{tabular}{c|cccc|rccc|rccc} 
& $y^{3} y^{6}$ & $y^{4} \bar{y}^{4}$ & $y^{5} \bar{y}^{5}$ & $\bar{y}^{3} \bar{y}^{6}$ & \multicolumn{1}{|c|}{$y^{1} \omega^{6}$} & $y^{2} \bar{y}^{2}$ & $\omega^{5} \bar{\omega}^{5}$ & $\bar{y}^{1} \bar{\omega}^{6}$ & $\omega^{1} \omega^{3}$ & $\omega^{2} \bar{\omega}^{2}$ & $\omega^{4} \bar{\omega}^{4} \bar{\omega}^{1} \bar{\omega}^{3}$ \\
\hline \hline $\mathbf{b}_{4}$ & 1 & 0 & 0 & 1 & 0 & 0 & 1 & 0 & 0 & 0 & 1 & 0 \\
$\beta$ & 0 & 0 & 0 & 1 & 0 & 1 & 0 & 1 & 1 & 0 & 1 & 0 \\
$\gamma$ & 0 & 0 & 1 & 1 & 1 & 0 & 0 & 1 & 0 & 1 & 0 & 0
\end{tabular}

The choice of generalized GSO coefficients is:

$$
\begin{gathered}
c\left(\begin{array}{c}
\mathbf{b}_{4} \\
\mathbf{b}_{j}, \beta
\end{array}\right) \quad=-c\left(\begin{array}{c}
\mathbf{b}_{4} \\
\mathbf{1}
\end{array}\right)=-c\left(\begin{array}{c}
\beta \\
\mathbf{1}
\end{array}\right)=c\left(\begin{array}{c}
\beta \\
\mathbf{b}_{j}
\end{array}\right)= \\
-c\left(\begin{array}{c}
\beta \\
\gamma
\end{array}\right) \quad=c\left(\begin{array}{c}
\gamma \\
\mathbf{b}_{2}
\end{array}\right)=-c\left(\begin{array}{c}
\gamma \\
\mathbf{b}_{1}, \mathbf{b}_{3}, \mathbf{b}_{4}, \gamma
\end{array}\right)=-1
\end{gathered}
$$

$(j=1,2,3)$, with the others specified by modular invariance and space-time supersymmetry. A property of the FNY model that distinguishes it from the NAHEbased models of refs. [8, 13] is the choice of pairings of the left- and right-moving real fermions from the set $\{y, \omega \mid \bar{y}, \bar{\omega}\}^{1, \cdots, 6}$. With the choice of pairing in FNY, the three left-moving pairs $y^{3} y^{6}, y^{1} \omega^{6}$ and $\omega^{1} \omega^{3}$ are complexified, and the remaining left-moving real fermions from the set $\{y, \omega\}^{1, \cdots, 6}$ are paired with right-moving real fermions to produce Ising model operators. In the right-moving sector 19 worldsheet fermions are complex and generate the observable and hidden four dimensional gauge groups. We remark that both the choice of pairings as well as that of the GSO phases affect nontrivially the spectrum of the string models, and consequently their specific phenomenological characteristics [14]. This is an important remark, keeping in mind that the eventual goal of string theory is to learn how a particular vacuum is selected dynamically.

\section{$2.2 \quad$ Gauge group}

The gauge group before imposing the flat directions consist of the universal observable $S O(10)$ sub-group, $S U(3)_{C} \times S U(2)_{L} \times U(1)_{C} \times U(1)_{L}$, generated by the five complex world-sheet fermions $\bar{\psi}^{1, \cdots, 5}$; six observable horizontal, flavor-dependent, $U(1)$ symmetries $U(1)_{1, \cdots, 6}$, generated by $\left\{\bar{\eta}^{1}, \bar{\eta}^{2}, \bar{\eta}^{3}, \bar{y}^{3} \bar{y}^{6}, \bar{y}^{1} \bar{\omega}^{6}, \bar{\omega}^{1} \bar{\omega}^{3}\right\}$, respectively; and the unbroken sub-group $S O(4) \times S U(3) \times U(1)^{4}$ of the hidden $E_{8}$, generated by $\bar{\phi}^{1, \cdots, 8}$. The weak hypercharge is given by

$$
U(1)_{Y}=\frac{1}{3} U(1)_{C} \pm \frac{1}{2} U(1)_{L}
$$

where switching signs in eq. (2.3) corresponds to flipping of the representations,

$$
\begin{aligned}
+ & \leftrightarrow- \\
e_{L}^{c} & \leftrightarrow N_{L}^{c} \\
u_{L}^{c} & \leftrightarrow d_{L}^{c}
\end{aligned}
$$

This flip is equivalent to the flip between the straight and flipped $S U(5)$ representations [15]. In the case of $S U(5)$ only the later choice is allowed as there are no 
adjoint representations to break the non-Abelian gauge symmetry in the former. In the case of the standard-like models, as the GUT non-Abelian symmetry is broken directly at the string level, this flip is consistent with the requirement that the gauge group can be broken to the Standard Model gauge group in the effective low energy field theory. We also note that under this $Z_{2}$ flip the Higgs representations are also flipped $h \leftrightarrow \bar{h}$.

In the following we will show that the choice of the sign in eq. (2.3) also has interesting consequences in terms of the decoupling of the exotic fractionally charged states. The other combination of $U(1)_{C}$ and $U(1)_{L}$, which is orthogonal to $U(1)_{Y}$ is given by

$$
U(1)_{Z^{\prime}}=U(1)_{C} \mp U(1)_{L}
$$

We will show that the choices of flat directions in the model of ref. [2] forces either $U(1)_{Y}$ or $U(1)_{Z^{\prime}}$ to be broken. Therefore, in the phenomenologically viable case we are forced to have only $S U(3)_{C} \times S U(2)_{L} \times U(1)_{Y}$ as the unbroken $S O(10)$ subgroup below the string scale. While we have no dynamical mechanism that chooses between these two distinct vacua, we find it to be an interesting example of how string dynamics may force the $S O(10)$ subgroup below the string scale to coincide with the Standard Model gauge group.

\subsection{Matter spectrum}

The full massless spectrum of the model, together with the quantum numbers under the right-moving gauge group, are given in ref. [2]. Here we give a brief summary. The sectors $\mathbf{b}_{1}, \mathbf{b}_{2}$ and $\mathbf{b}_{3}$ correspond to the three twisted sectors of the $Z_{2} \times Z_{2}$ orbifold model and produce three generations in the 16 representation of $S O(10)$ decomposed under $S U(3)_{C} \times S U(2)_{L} \times U(1)_{C} \times U(1)_{L}$, with charges under the horizontal symmetries.

The Neveu-Schwarz (NS) sector corresponds to the untwisted sector and produces in addition to the gravity and gauge multiplets three pairs of electroweak scalar doublets $\left\{h_{1}, h_{2}, h_{3}, \bar{h}_{1}, \bar{h}_{2}, \bar{h}_{3}\right\}$, seven pairs of $S O(10)$ singlets with observable $U(1)$ charges, $\left\{\phi_{12}, \bar{\phi}_{12}, \phi_{23}, \bar{\phi}_{23}, \phi_{13}, \bar{\phi}_{13}, \phi_{56}, \bar{\phi}_{56}, \phi_{56}^{\prime}, \bar{\phi}_{56}^{\prime}, \phi_{4}, \bar{\phi}_{4}, \phi_{4}^{\prime}, \bar{\phi}_{4}^{\prime}\right\}$, and three scalars that are singlets of the entire four dimensional gauge group, $\phi_{1}, \phi_{2}, \phi_{3}$.

In the model of ref. [2] the states from the NS sector and the sectors $\mathbf{b}_{1}, \mathbf{b}_{2}$ and $\mathbf{b}_{3}$ are the only ones that transform solely under the observable, $S U(3)_{C} \times S(2)_{L} \times$ $U(1)_{C} \times U(1)_{L} \times U(1)_{1, \cdots, 6}$ gauge group. The choice of GSO phase $c\left(\mathbf{b}_{4}, 1\right)=+1$ projects all the massless states from the sector $\mathbf{b}_{4}$ as well as the space-time vector bosons from the sector $\mathbf{I}=\mathbf{1}+\mathbf{b}_{1}+\mathbf{b}_{2}+\mathbf{b}_{3}$.

The sectors $\mathbf{b}_{j}+2 \gamma$ and $\mathbf{b}_{j}+2 \gamma+\mathbf{I}$ with $j=1, \cdots, 4$ produce $S O(10)$ singlet matter states in the 16 vector representation of the hidden $S O(16)$ gauge group, decomposed under the final hidden group and are listed in Table I. The sectors with some combination of $\left\{\mathbf{1}, \mathbf{b}_{1}, \mathbf{b}_{2}, \mathbf{b}_{3}, \mathbf{b}_{4}, \beta\right\}$ plus $\gamma$ produce states that are $S U(3)_{C} \times$ $S U(2)_{L}$ singlets, but are charged under $U(1)_{Y}$ or $U(1)_{Z^{\prime}}$. Similar states arise also 
from the sectors $\mathbf{1}+\mathbf{b}_{1}+\beta+2 \gamma$ and $\mathbf{I}+\beta+2 \gamma$. These states arise due to the breaking of the $S O(10)$ symmetry, by the basis vectors $\beta$ and $\gamma$, and carry fractional electric charge $\pm 1 / 2$ or fractional $U(1)_{Z}$, charge. These are exotic stringy states that do not fall into representations of the original $S O(10)$ symmetry. One of the important goals of our paper is to find the flat $F$ and $D$ solutions that give heavy mass to all the exotic fractionally charged states.

The trilinear superpotential of the string model is given by $W=\sqrt{2} g\left[W_{1}+W_{2}\right]$ where,

$$
\begin{aligned}
W_{1}= & \left\{\left(u_{L_{1}}^{c} Q_{1}+N_{L_{1}}^{c} L_{1}\right) \bar{h}_{1}+\left(d_{L_{2}}^{c} Q_{2}+e_{L_{2}}^{c} L_{2}\right) h_{2}+\left(d_{L_{3}}^{c} Q_{3}+e_{L_{3}}^{c} L_{3}\right) h_{3}\right. \\
& +h_{2} \bar{h}_{1} \bar{\phi}_{12}+\bar{h}_{2} h_{1} \phi_{12}+h_{3} \bar{h}_{1} \bar{\phi}_{13}+\bar{h}_{3} h_{1} \phi_{13}+h_{3} \bar{h}_{2} \phi_{23}+\bar{h}_{3} h_{2} \bar{\phi}_{23} \\
& \left.+\phi_{12} \bar{\phi}_{13} \bar{\phi}_{23}+\bar{\phi}_{12} \phi_{13} \phi_{23}+\left(\phi_{4} \bar{\phi}_{4}^{\prime}+\bar{\phi}_{4} \phi_{4}^{\prime}\right) \phi_{1}\right\} \\
W_{2}= & \frac{1}{\sqrt{2}}\left\{H_{1} H_{2} \phi_{4}+\left(H_{3} H_{4}+H_{5} H_{6}\right) \bar{\phi}_{4}+\left(H_{7} H_{8}+H_{9} H_{10}\right) \phi_{4}^{\prime}+H_{11} H_{13} \bar{\phi}_{4}^{\prime}\right. \\
& \left.+\left(V_{41} V_{42}+V_{43} V_{44}\right) \bar{\phi}_{4}+V_{45} V_{46} \phi_{4}+\left(V_{47} V_{48}+V_{49} V_{50}\right) \bar{\phi}_{4}^{\prime}+V_{51} V_{52} \phi_{4}^{\prime}\right\} \\
& +\left\{H_{15} H_{16} \phi_{56}^{\prime}+\left(H_{17} H_{18}+H_{21} H_{22}\right) \bar{\phi}_{56}+H_{19} H_{20} \bar{\phi}_{56}^{\prime}\right\} \\
& +\left(V_{11} V_{12}+V_{13} V_{14}+V_{15} V_{17}+V_{19} V_{20}\right) \phi_{13} \\
& +\left(V_{21} V_{22}+V_{23} V_{24}+V_{25} V_{27}+V_{29} V_{30}\right) \phi_{12} \\
& +V_{31} V_{32} \bar{\phi}_{23}+V_{33} V_{34} \phi_{23}+H_{29} H_{30} \bar{\phi}_{13}+H_{36} H_{37} \phi_{12} \\
& +\left\{\left(H_{31} H_{34}+V_{43} V_{45}\right) h_{2}+V_{44} V_{46} \bar{h}_{2}+V_{47} V_{52} h_{3}+\left(H_{38} H_{41}+V_{48} V_{51}\right) \bar{h}_{3}\right\} .
\end{aligned}
$$

\subsubsection{Abelian anomaly}

With the choice of GSO projection coefficients in eq. (2.2) the following $U(1)$ symmetries (from the entire set of $12\left\{U_{C}, U_{L}, U_{i=1, \text { to } 6}, U_{j=H, 7,8,9}\right\}$ ) are anomalous: $\operatorname{Tr} U_{1}=-24, \operatorname{Tr} U_{2}=-30, \operatorname{Tr} U_{3}=18, \operatorname{Tr} U_{5}=6, \operatorname{Tr} U_{6}=6$ and $\operatorname{Tr} U_{8}=12$. The total anomaly from all six of these Abelian symmetries can be rotated into a single $U(1)_{\mathrm{A}}$, uniquely defined by

$$
U(1)_{\mathrm{A}} \equiv c_{A} \sum_{i}\left\{\operatorname{Tr} Q_{i}^{(A)}\right\} U(1)_{i},
$$

with $c_{A}$ a normalization coefficient. In this case the single anomalous Abelian symmetry becomes,

$$
U_{A} \equiv-4 U_{1}-5 U_{2}+3 U_{3}+U_{5}+U_{6}+2 U_{8}
$$

After this rotation, the universal Green-Schwarz relation invoked by modular invariance constraints removes all Abelian triangle anomalies except those involving either one or three $U_{A}$ gauge bosons. That is, the five orthogonal, anomaly-free, Abelian combinations involving $U_{1,2,3,5,6,8}$, together with the other six anomaly free $U(1)$ 's are free of all gauge and mixed trilinear anomalies. 
The standard anomaly cancellation mechanism [6] breaks $U_{A}$ and in the process generates an FI $D$-term,

$$
\epsilon \equiv \frac{g_{s}^{2} M_{P}^{2}}{192 \pi^{2}} \operatorname{Tr} Q_{A}
$$

where $g_{s}$ is the string coupling and $M_{P}$ is the reduced Planck mass, $M_{P} \equiv$ $M_{\text {Planck }} / \sqrt{8 \pi} \approx 2.4 \times 10^{18} \mathrm{GeV}$. Spacetime supersymmetry is broken near the string scale by the FI $D_{A}$-term unless a set of scalar VEVs, $\left\{\left\langle\varphi_{m}\right\rangle\right\}$, carrying anomalous charges $Q_{m}^{(A)}$ can contribute a compensating $\left\langle D_{A}\left(\varphi_{m}\right)\right\rangle \equiv \sum_{\alpha} Q_{m}^{(A)}\left|\left\langle\varphi_{m}\right\rangle\right|^{2}$ term to cancel the FI term, i.e.,

$$
\left\langle D_{A}\right\rangle=\sum_{m} Q_{m}^{(A)}\left|\left\langle\varphi_{m}\right\rangle\right|^{2}+\epsilon=0
$$

thereby restoring supersymmetry. A set of scalar VEVs satisfying eq. (2.11) is also constrained to keep $D$-flatness for all non-anomalous Abelian $U_{i}$ symmetries as well,

$$
\left\langle D_{i}\right\rangle=\sum_{m} Q_{m}^{(i)}\left|\left\langle\varphi_{m}\right\rangle\right|^{2}=0
$$

Each superfield $\Phi_{m}$ (containing a scalar field $\varphi_{m}$ and superpartner) in the superpotential imposes further constraints on the scalar VEVs. F-flatness will be broken (thereby destroying spacetime supersymmetry) at the scale of the VEVs unless,

$$
\left\langle F_{m}\right\rangle=\left\langle\frac{\partial W}{\partial \Phi_{m}}\right\rangle=0 ;\langle W\rangle=0 .
$$

\subsubsection{Fractionally charged states}

By examining the fractionally charged states and the trilinear superpotential, it is seen that all the fractionally charged states receive a heavy mass by giving a VEV to the neutral singlets $\bar{\phi}_{4}, \bar{\phi}_{4}^{\prime}, \phi_{4}, \phi_{4}^{\prime}[3]$. The additional trilinear terms, $\left(\phi_{4} \bar{\phi}_{4}^{\prime}+\bar{\phi}_{4} \phi_{4}^{\prime}\right) \phi_{1}$ impose an $F$-flatness constraint from eq. (2.13),

$$
\left(\left\langle\phi_{4}\right\rangle\left\langle\bar{\phi}_{4}^{\prime}\right\rangle+\left\langle\bar{\phi}_{4}\right\rangle\left\langle\phi_{4}^{\prime}\right\rangle\right)=0 \text {. }
$$

A $D$-flat solution to eqs. (2.11) and (2.12) which also satisfies the $F$-flatness constraints eq. (2.13) (and (2.14) in particular) is given, for example, by the following set of fields

$$
\left\{\phi_{12}, \phi_{23}, \bar{\phi}_{56}, \phi_{4}, \phi_{4}^{\prime}, \bar{\phi}_{4}, \bar{\phi}_{4}^{\prime}, H_{15}, H_{30}, H_{31}, H_{38}\right\}
$$

\footnotetext{
${ }^{\dagger}$ Based on the arguments of [16], $M$-theory does not appear to alter the form of the FI term. Instead an $M$-theory FI term should remain identical to the FI term obtained for a weakly-coupled $E_{8} \times E_{8}$ heterotic string, independent of the size of $M$-theory's 11th dimension.

${ }^{\ddagger}$ Here we consider flat directions involving only non-Abelian singlet fields. In cases where nontrivial non-Abelian representations are also allowed to take on VEVs, generalized non-Abelian $D$-flat constraints must also be imposed.
} 
with the VEVs

$$
\begin{aligned}
& \begin{aligned}
\frac{1}{3}\left|\left\langle\phi_{12}\right\rangle\right|^{2} & =\left|\left\langle\phi_{23}\right\rangle\right|^{2}=\left|\left\langle\bar{\phi}_{56}\right\rangle\right|^{2}=\frac{1}{2}\left|\left\langle H_{15}\right\rangle\right|^{2}=\frac{1}{3}\left|\left\langle H_{30}\right\rangle\right|^{2}=\left|\left\langle H_{31}\right\rangle\right|^{2}=\frac{1}{2}\left|\left\langle H_{38}\right\rangle\right|^{2} \\
& \equiv|\langle\alpha\rangle|^{2}
\end{aligned} \\
& \left(\left|\left\langle\phi_{4}\right\rangle\right|^{2}+\left|\left\langle\phi_{4}^{\prime}\right\rangle\right|^{2}\right)-\left(\left|\left\langle\bar{\phi}_{4}\right\rangle\right|^{2}+\left|\left\langle\bar{\phi}_{4}^{\prime}\right\rangle\right|^{2}\right)=|\langle\alpha\rangle|^{2}
\end{aligned}
$$

where $\langle\alpha\rangle$ is an overall scale (assumed to be real)

Cancellation of the FI term by this $D$-flat solution specifies the scale $\langle\alpha\rangle$. Inserting the flat direction VEVs and anomalous charge trace in (2.11) yields,

$$
\left\langle D_{\mathrm{A}}\right\rangle=-224|\langle\alpha\rangle|^{2}+\frac{g_{s}^{2} M_{P}^{2} 1344}{192 \pi^{2}}=0 .
$$

At $M_{U}=2.5 \times 10^{16} \mathrm{GeV}$, the unified couplings have a value $\alpha_{U} \equiv g_{\text {physical }}^{2} /(4 \pi) \approx$ $1 / 24$. This corresponds to $g_{s} \approx .5$, since $g_{\text {physical }}=\sqrt{2} g_{s} \approx .72$. Thus, the FI scale for the FNY model is

$$
|\langle\alpha\rangle| \approx 7 \times 10^{16} \mathrm{GeV} .
$$

In ref. [17], the space of $F$ - and $D$-flat directions that decouple the fractionally charged states will be studied in more detail. The following important remarks are, however, in order. Following the holomorphic gauge-invariant polynomial method developed in refs. [9] and [4] or the matrix method of ref. [10], it is possible to classify the complete space of allowed $D$-flat directions. When the non-Abelian singlet fields allowed to take on VEVs are restricted to those that do not break the weak-hypercharge, it is observed that all the possible $D$-flat solutions (that cancel the FI term) contain fields that break the $U(1)_{Z^{\prime}}$ symmetry, eq. (2.5). In other words, no directions that are $D$-flat for all non-anomalous Abelian symmetries and are formed solely from non-Abelian singlet fields uncharged under both $U(1)_{Y}$ and $U(1)_{Z^{\prime}}$, make a contribution to the anomalous $D$-term that can cacell the FI-term. Specifically, anomalous $D$-term cotribution is 0 for all of these direction.

We then have the very interesting situation in which the $U(1)_{Z^{\prime}}$ is necessarily broken in the $D$-flat string vacuum. The FNY model therefore presents the first example where the string consistency constraints force the $S O(10)$ symmetry to be broken to $S U(3)_{C} \times S U(2)_{L} \times U(1)_{Y}$ by singlet VEVs, rather than $S U(3) \times S U(2)_{L} \times$ $U(1)_{C} \times U(1)_{L}$. Possible generalization of this constraint for VEVs of non-Abelian states will be examined in [17].

\footnotetext{
$\S$ Together eqs. (2.14) and (2.16) still allow degrees of freedom within the VEVs of the $\left\{\phi_{4}, \phi_{4}^{\prime}, \bar{\phi}_{4}, \bar{\phi}_{4}^{\prime}\right\}$ fields. VEV scales of orders lower than $|\langle\alpha\rangle|$ for these fields would imply some mass scales of exotics being below the FI scale $|\langle\alpha\rangle|$. However, naturalness of values would suggest that the VEVs of all of these fields should be of the same scale as $|\langle\alpha\rangle|$.

IThis flat direction is formed from a combination of VEVs, denoted $M_{6}, M_{7}$ and $R_{10}$ in [4]. $M_{6}, M_{7}$ and $R_{10}$ are individually $D$-flat for each non-anomalous $U(1)$ and $R_{10}$ is responsible for cancelling the FI term.
} 
We should remark, however, that we also anticipate that there exist, phenomenologically unviable, $D$-flat singlet solutions in which the weak-hypercharge is broken and the $U(1)_{Z^{\prime}}$ remains unbroken. The existence of such solutions will also be investigated in ref. [17]. At present we do not know what is the mechanism, or the reason, that selects the $U(1)_{Y}$-preserving vacuum over the $U(1)_{Z}^{\prime}$-preserving vacuum.

The $D$-flat direction (2.16) was found to be $F$-flat to all orders in the stringbased superpotential [17]. F-flatness is broken in a field-theoretic gauge-invariant superpotential by generic terms of the form

$$
\begin{array}{ll}
\left(\phi_{1,2,3}\right)^{m=0,1}\left(\phi_{4} \bar{\phi}_{4}\right)^{n} \quad ; & \left(\phi_{1,2,3}\right)^{m=0,1}\left(\phi_{4}^{\prime} \bar{\phi}_{4}^{\prime}\right)^{n} ; \\
\left(\phi_{1,2,3}\right)^{m=0,1}\left(\phi_{4} \bar{\phi}_{4}^{\prime}\right)^{n} \quad ; \quad & \left(\phi_{1,2,3}\right)^{m=0,1}\left(\phi_{4}^{\prime} \bar{\phi}_{4}\right)^{n},
\end{array}
$$

where $n$ is any positive integer. However, string world-sheet selection rules impose strong constraints on allowed superpotential terms beyond gauge invariance. Here, for example, all (2.19) terms are forbidden by the requirement that no more than $N-4$ (for $N \geq 4$ ) NS fields can appear in an $N^{\text {th }}$ order term [19, 18]. All fields in (2.19) are NS class.

The next observation we wish to make is to recall that we have a $Z_{2}$ ambiguity in the definition of the weak-hypercharge, eq. (2.3). Choosing the positive sign imposes the decoupling condition on the fractionally charged states, eq. (2.14). Choosing the negative sign in the definition of the weak-hypercharge, eq. (2.3), flips between the exotic fractionally charged states and the electrically neutral exotic states with fractional $U(1)_{Z^{\prime}}$ charge. There is a class of exotic states from the sectors $\mathbf{1}+\mathbf{b}_{1}+$ $\beta+2 \gamma$ and $\mathbf{I}+\beta+2 \gamma$ which is invariant under this flip. These states do not have $U(1)_{C}$ charge and are either $S U(2)_{L}$ singlets with $U(1)_{L}= \pm 1$, or $S U(2)_{L}$ doublets with $U(1)_{L}=0$. In both cases they carry fractional electric charge $\pm 1 / 2$. With the positive sign in the weak-hypercharge definition all the states with fractional electric charge gain mass at the cubic level of the superpotential by the VEVs of $\phi_{4}, \phi_{4}^{\prime}, \bar{\phi}_{4}, \bar{\phi}_{4}^{\prime}$. With the negative sign definition of the weak-hypercharge the states $\left\{H_{15}, H_{16}, H_{17}, H_{18}, H_{19}, H_{20}, H_{21}, H_{22}, H_{23}, H_{24}, H_{25}, H_{26}, H_{27}, H_{28}\right\}$ in Table 2 of ref. [2], carry fractional electric charge $\pm 1 / 2$ and are not coupled to $\phi_{4}, \phi_{4}^{\prime}, \bar{\phi}_{4}, \bar{\phi}_{4}^{\prime}$ at the cubic level of the superpotential. The states from the sectors $\mathbf{1}+\mathbf{b}_{1}+\beta+2 \gamma$ and $\mathbf{I}+\beta+2 \gamma$ are, of course, still electrically charged and can receive heavy mass from the VEVs of $\phi_{4}, \phi_{4}^{\prime}, \bar{\phi}_{4}, \bar{\phi}_{4}^{\prime}$. We can contemplate other $F$-flat and $D$-flat solutions that will make all the fractionally charged states super-heavy. However, we see that with the negative sign definition of the weak-hypercharge this will be far more difficult to achieve. The discussion above illustrates how also the $Z_{2}$ ambiguity in the weakhypercharge definition is broken by the choices of $F$ - and $D$-flat solutions.

\subsubsection{Exotic triplet/anti-triplet pair}


The massless spectrum of the FNY model contains in addition to the exotic fractionally charged states, one pair of $S U(3)_{C}$ triplets in vector-like representation $\left(H_{33}, H_{40}\right)$ with fractional $U(1)_{Z^{\prime}}$ charge and several pairs of electroweak Higgs doublets. To show that with the $F$ - and $D$-flat solution, eq. (2.16), the spectrum below the string scale indeed coincides with that of the MSSM, we have to show that in this vacuum these additional states receive mass near the string scale, with only one pair of Higgs doublets remaining light.

A mass term,

$$
m_{\text {trip }}=\frac{A_{5}\left\langle\phi_{23}\right\rangle\left\langle H_{38}\right\rangle\left\langle H_{31}\right\rangle}{M_{S}^{2}}=\langle\alpha\rangle \frac{\sqrt{2} A_{5}\langle\alpha\rangle^{2}}{M_{S}^{2}},
$$

for the additional color triplet pair appears from the quintic superpotential term,

$$
H_{33} H_{40} H_{31} H_{38} \phi_{23}
$$

While this triplet mass is generated at the FI scale $=\langle\alpha\rangle \approx 7 \times 10^{16} \mathrm{GeV}$, we estimate the additional factor in $m_{\text {trip }}$, which includes the five-point string amplitude $A_{5}$ (defined here to not include the $1 / M_{S}^{2}$ factor) for this specific fifth-order nonrenormalizable coupling, will contribute a suppression factor on the order of $\sim(1 / 10-1 / 100)$. The five-point string amplitude includes a world-sheet integral $I_{2}$. Similar worldsheet integrals have been computed for other fifth order superpotential terms, both in different NAHE- [18 and non-NAHE-based [20, 21] free fermionic models. The other worldsheet integral values were all found to be of the same order and our estimate of $m_{\text {trip }}$ here assumes our $I_{2}$ value is comparable to those others. (Note that the triplet mass "suppression factor" $\left(\sqrt{2} A_{5}\langle\alpha\rangle / M_{S}\right)^{2}$ would appear to be of order one.) While the exotic triplet mass may lie slightly below the string/unification scale of $M_{U}=2.5 \times 10^{16} \mathrm{GeV}$, it appears sufficiently close to $M_{U}$ so as not to significantly affect the running of the MSSM couplings. We emphasize that the numerical estimate of the masses arising from the singlet VEVs should be regarded only as illustrative. The important result is the generation of mass terms for all the states beyond the MSSM, near the string scale. The actual masses of the extra fields may be spread around the $M_{U}$ scale, thus inducing small threshold corrections that are still expected to be compatible with the low energy experimental data.

\subsubsection{Exotic doublets}

We next turn to the analysis of the Higgs mass spectrum generated by the $F$ - and $D$-flat solution eq. (2.16). The FNY model contains four $h_{u p}$-class MSSM $S U(2)$ doublets

$$
\left\{\bar{h}_{1}, \bar{h}_{2}, \bar{h}_{3}, \bar{h}_{4} \equiv H_{34}\right\}
$$

and four $h_{\text {down }}$-class doublets,

$$
\left\{h_{1}, h_{2}, h_{3}, h_{4} \equiv H_{41}\right\} .
$$


A mass matrix $M$ yielding doublet mass terms,

$$
\left(h_{1}, h_{2}, h_{3}, h_{4}\right) M\left(\bar{h}_{1}, \bar{h}_{2}, \bar{h}_{3}, \bar{h}_{4}\right)^{T}
$$

results from the flat direction VEVs. This mass matrix

$$
M_{h_{i}, \bar{h}_{j}}=\left(\begin{array}{cccc}
0 & g\left\langle\phi_{12}\right\rangle & 0 & 0 \\
0 & 0 & 0 & g\left\langle H_{31}\right\rangle \\
0 & g\left\langle\phi_{23}\right\rangle & 0 & 0 \\
0 & 0 & g\left\langle H_{38}\right\rangle & \frac{A_{5}\left\langle\phi_{23}\right\rangle\left\langle H_{38}\right\rangle\left\langle H_{31}\right\rangle}{M_{s}^{2}}
\end{array}\right)
$$

with $\left\langle\phi_{12}\right\rangle=\sqrt{3}\langle\alpha\rangle,\left\langle\phi_{23}\right\rangle=\langle\alpha\rangle,\left\langle H_{31}\right\rangle=\langle\alpha\rangle$, and $\left\langle H_{38}\right\rangle=\sqrt{2}\langle\alpha\rangle$. The zeros in the matrix hold to all orders in the stringy superpotential. This can be proven simply by gauge-invariance constraints and the $N-4$ NS rule. The NS rule eliminates three non-zero mass terms, that would appear in a field-theoretic model:

$$
\begin{aligned}
& M_{h_{1} \bar{h}_{4}}=\left\langle\phi_{12} H_{31}\right\rangle A_{4} / M_{s} ; \quad M_{h_{3} \bar{h}_{4}}=\left\langle\phi_{23} H_{31}\right\rangle A_{4}^{\prime} / M_{s} ; \\
& M_{h_{4} \bar{h}_{2}}=\left\langle\phi_{23} H_{38}\right\rangle A_{4}^{\prime \prime} / M_{s} .
\end{aligned}
$$

The field-theoretic $M_{h_{1} \bar{h}_{4}}$ and $M_{h_{3} \bar{h}_{4}}$ values appear in the same ratio as $M_{h_{1} \bar{h}_{2}}$ and $M_{h_{3} \bar{h}_{2}}$ (provided $A_{4}=A_{4}^{\prime}$ ). Hence, we note that the field-theoretic and string models involve the same eigenstates and eigenmasses of $h_{1}$ and $h_{3}$.

To determine the $h$ and $\bar{h}$ mass eigenstates and eigenvalues we evaluate the eigenstates and eigenvalues of $M M^{\dagger}$ and $M^{\dagger} M$ respectively. We find there is exactly one massless Higgs-like eigenvalue pair:

$$
h_{3}^{\prime}=\frac{1}{2}\left(-h_{1}+\sqrt{3} h_{3}\right) \quad \text { and } \quad \bar{h}_{1} \quad \text { with } \quad m_{h_{3}^{\prime}}^{2}=m_{\bar{h}_{1}}^{2}=0 ;
$$

and three pairs with string scale masses:

$$
\begin{aligned}
h_{1}^{\prime} & =\frac{1}{2}\left(\sqrt{3} h_{1}+h_{3}\right) \quad \text { and } \bar{h}_{2} \quad \text { with } m_{h_{1}^{\prime}}^{2}=m_{\bar{h}_{2}}^{2}=4 g^{2}\langle\alpha\rangle^{2} ; \\
h_{2}^{\prime} & =c_{2^{\prime}}\left[-\left(A^{2}+B^{2}+\sqrt{A^{4}+6 A^{2} B^{2}+B^{4}}\right) h_{2}+(2 A B) h_{4}\right] \text { and } \\
\bar{h}_{4}^{\prime} & =\bar{c}_{4^{\prime}}\left[(2 \sqrt{2} A B) \bar{h}_{3}-\left(A^{2}-B^{2}+\sqrt{A^{4}+6 A^{2} B^{2}+B^{4}}\right) \bar{h}_{4}\right] \quad \text { with } \\
m_{h_{2}^{\prime}}^{2} & =m_{\bar{h}_{4}^{\prime}}^{2}=\left(3 A^{2}+B^{2}-\sqrt{-8 A^{4}+\left(3 A^{2}+B^{2}\right)^{2}}\right) / 2 ; \\
h_{4}^{\prime} & =c_{2^{\prime}}\left[(2 A B) h_{2}+\left(A^{2}+B^{2}+\sqrt{A^{4}+6 A^{2} B^{2}+B^{4}}\right) h_{4}\right] \quad \text { and } \\
\bar{h}_{3}^{\prime} & =\bar{c}_{4^{\prime}}\left[\left(A^{2}-B^{2}+\sqrt{A^{4}+6 A^{2} B^{2}+B^{4}}\right) \bar{h}_{3}+(2 \sqrt{2} A B) \bar{h}_{4}\right] \text { with } \\
m_{h_{4}^{\prime}}^{2} & =m_{\bar{h}_{3}^{\prime}}^{2}=\left(3 A^{2}+B^{2}+\sqrt{-8 A^{4}+\left(3 A^{2}+B^{2}\right)^{2}}\right) / 2 ;
\end{aligned}
$$

where $A \equiv g\langle\alpha\rangle, B \equiv \frac{\sqrt{2} A_{5}\langle\alpha\rangle^{3}}{M_{s}^{2}}$, and $c_{2^{\prime}}$ and $\bar{c}_{4}$ are normalization constants. 
Thus, $h_{2}^{\prime}, \bar{h}_{3}^{\prime}, h_{4}^{\prime}$, and $\bar{h}_{3}^{\prime}$ obtain masses of $\mathcal{O}(\langle\alpha\rangle)$, along with the fractionally charged states. Note that the Higgs spectrum is simplified in the $B<<A$ limit, (i.e., no mass mixing term between $H_{41}$ and $H_{35}$ ), the $h_{2,4}$ and $\bar{h}_{3,4}$ mass eigenstates and eigenvalues reduce to

$$
\begin{aligned}
& h_{2} \quad \text { and } \quad \bar{h}_{4} \quad \text { with } \quad m_{h_{2}}^{2}=m_{\bar{h}_{4}}^{2}=A^{2} \equiv g^{2}|\langle\alpha\rangle|^{2} \\
& h_{4} \quad \text { and } \quad \bar{h}_{3} \quad \text { with } \quad m_{h_{4}}^{2}=m_{\bar{h}_{3}}^{2}=2 A^{2} \equiv 2 g^{2}|\langle\alpha\rangle|^{2} \text {. }
\end{aligned}
$$

Hence, consistent with MSSM physics, we see that the flat direction of eq. (2.16) produces in the low energy effective field theory a single pair of massless Higgs doublets above the supersymmetry breaking scale.

\subsubsection{Exotic singlets \& hidden sector states}

The FNY model contains 16 non-Abelian singlet states with fractional electric charge, 44 hypercharge-neutral singlets, and three uncharged states. All 16 fractionally charged states receive induced masses from renormalizable superpotential terms involving VEVs of (2.15). $14+1$ of the remaining $44+3$ singlets similarly receive masses from third order terms, while an additional four singlets receive mass at fifth order. $26+2$ singlets remain massless through at least sixth order.

Approximately two-thirds of the 34 non-Abelian hidden sector states become massive from third (all 4 fractionally charged states plus 8 other states), fourth (6 states), or fifth order terms (4 states). One $S U(3)_{H}$ triplet/anti-triplet pair, six $S U(2)_{H}$ doublets, and four $S U(2)_{H}^{\prime}$ doublets remain massless through at least sixth order.

The appearance of exotic singlets and hidden non-Abelian states uncharged under the SM, still has significant phenomenological implications. In particular, when such states appear in a string model, we should expect them to interact with SM states via the extra $U(1)$ charges. Surprisingly, for the particular flat direction considered we find that, after decoupling of the massive states from the low energy theory, no such interactions appearing in the effective Yukawa produced by the third order nonrenomalizable superpotential and at least fourth through sixth order superpotential terms ${ }^{\|}$containing one to three VEVs, respectively. Interestingly, all hidden sector terms in the effective Yukawa appear with $\langle\alpha\rangle / M_{s}$ factors. That is, all hidden sector "non-suppressed" third order terms are removed when massive states are decoupled.

$$
\begin{aligned}
& W_{(M S S M)}: g_{s} \sqrt{2} \bar{h}_{1}\left[Q_{1} u_{1}^{c}+L_{1} N_{1}^{c}\right]+g_{s} \sqrt{2} \cos \sqrt{3} h_{3}^{\prime}\left[Q_{3} d_{3}^{c}+L_{3} e_{3}^{c}\right] \\
& W_{(\text {Singlet })} \quad: \quad g_{s} \sqrt{2} \phi_{1}\left[\phi_{4} \bar{\phi}_{4}^{\prime}+\phi_{4}^{\prime} \bar{\phi}_{4}\right]+g_{s} \sqrt{2} \bar{\phi}_{56}^{\prime} H_{19} H_{20}+g_{s} \sqrt{2} \bar{\phi}_{23} V_{31} V_{32} \\
& W_{(\text {Hidden })}: \lambda_{4} \frac{<\alpha>}{M_{s}} H_{19} V_{19} V_{37}+\lambda_{4}^{\prime} \frac{\sqrt{3}<\alpha>}{M_{s}} V_{31} H_{25} V_{35}+
\end{aligned}
$$

\footnotetext{
"Higher order contributions will be investigated in 17 .
} 


$$
\lambda_{5} \frac{<\bar{\phi}_{4}^{\prime}><\alpha>}{M_{s}^{2}} H_{19} V_{15} V_{40}+\lambda_{5}^{\prime} \frac{\sqrt{3}<\bar{\phi}_{4}^{\prime}><\alpha>}{M_{s}^{2}} V_{31} H_{23} V_{39}
$$

Effective Yukawa terms generated by third through sixth order superpotential terms containing one to three fields with VEVs, respectively.

\section{Comments}

Exotic MSSM states, many carrying fractional electric charge are a generic feature of many classes of string models. Most of these, if they remain massless down to the electroweak scale, signify unphysical phenomenology, thereby disallowing a model containing them. "String-selection rules" can make decoupling of dangerous exotic fields from the low energy effective field theory difficult. They often forbid several superpotential terms, otherwise allowed by gauge invariance, that could generate large mass for an exotic via couplings with flat direction VEVs [22].

Intermediate scale MSSM exotics are more phenomenologically viable than electroweak scale exotics. However, intermediate scale exotics will generally alter the running of the MSSM couplings and shift the unification scale away from the MSSM projected value of $M_{U} \approx 2.5 \times 10^{16} \mathrm{GeV}$. Further, one may argue that intermediate mass scales for MSSM exotics require additional ad hoc fine tuning, and therefore are not very attractive.

In this letter we have presented a string model wherein it is actually possible to decouple all SM-charged MSSM exotics from the effective field theory, giving mass to these fields at the Fayet-Iliopoulos (i.e., anomalous $U(1)$ ) scale, which is very near the string scale. Thus, we have found a string model consistent with both projected unification of the MSSM couplings at $M_{U} \approx 2.5 \times 10^{16} \mathrm{GeV}$ and the conjecture that the string scale $M_{S}$ may in fact coincide with $M_{U}$. This is the first string model that we are aware of with these properties.

The particular flat direction of the FNY model chosen herein is, in fact, not the model's only flat direction that decouples all MSSM exotics. A more complete set of flat directions that likewise perform this task will be presented in [17]. Detailed analysis of the physics of these different flat directions, including MSSM mass hierarchies, will be performed. Two difficulties with the example flat direction presented herein are that the flat direction does not yield an effective $\mu$ term at any order in the superpotential; and it gives (through at least sixth order terms) a top- $\nu_{\tau}$ universality without a seesaw mechanism, resulting in an $\mathcal{O}(100 \mathrm{GeV})$ neutrino. Our search for phenomenologically superior flat directions, more consistent with the MSSM, will be discussed in [17]. 


\section{Acknowledgments}

This work is supported in part by DOE Grants No. DE-FG-0294ER40823 (AF) and DE-FG-0395ER40917 (GC,DVN).

\begin{tabular}{|c|rrrrrrrrr|rrrrrrr|}
\hline State & $(C, L)$ & $Q_{C}$ & $Q_{L}$ & $Q_{1}$ & $Q_{2}$ & $Q_{3}$ & $Q_{4}$ & $Q_{5}$ & $Q_{6}$ & $S O(4)$ & $S U(3)$ & $Q_{H}$ & $Q_{7}$ & $Q_{8}$ & $Q_{9}$ \\
\hline \hline$V_{41}$ & $(1,1)$ & 0 & 1 & 0 & $\frac{1}{2}$ & 0 & $\frac{1}{2}$ & 0 & $-\frac{1}{2}$ & $(1,1)$ & $(1)$ & 0 & $\frac{1}{2}$ & 0 & $-\frac{1}{2}$ \\
$V_{42}$ & $(1,1)$ & 0 & -1 & 0 & $-\frac{1}{2}$ & 0 & $\frac{1}{2}$ & 0 & $\frac{1}{2}$ & $(1,1)$ & $(1)$ & 0 & $-\frac{1}{2}$ & 0 & $\frac{1}{2}$ \\
$V_{43}$ & $(1,1)$ & 0 & 1 & 0 & $-\frac{1}{2}$ & 0 & $\frac{1}{2}$ & 0 & $-\frac{1}{2}$ & $(1,1)$ & $(1)$ & 0 & $-\frac{1}{2}$ & 0 & $\frac{1}{2}$ \\
$V_{44}$ & $(1,1)$ & 0 & -1 & 0 & $\frac{1}{2}$ & 0 & $\frac{1}{2}$ & 0 & $\frac{1}{2}$ & $(1,1)$ & $(1)$ & 0 & $\frac{1}{2}$ & 0 & $-\frac{1}{2}$ \\
$V_{45}$ & $(1,2)$ & 0 & 0 & 0 & $-\frac{1}{2}$ & 0 & $-\frac{1}{2}$ & 0 & $\frac{1}{2}$ & $(1,1)$ & $(1)$ & 0 & $\frac{1}{2}$ & 0 & $-\frac{1}{2}$ \\
$V_{46}$ & $(1,2)$ & 0 & 0 & 0 & $\frac{1}{2}$ & 0 & $-\frac{1}{2}$ & 0 & $-\frac{1}{2}$ & $(1,1)$ & $(1)$ & 0 & $-\frac{1}{2}$ & 0 & $\frac{1}{2}$ \\
$V_{47}$ & $(1,1)$ & 0 & 1 & 0 & 0 & $\frac{1}{2}$ & $\frac{1}{2}$ & $\frac{1}{2}$ & 0 & $(1,1)$ & $(1)$ & 0 & $-\frac{1}{2}$ & 0 & $\frac{1}{2}$ \\
$V_{48}$ & $(1,1)$ & 0 & -1 & 0 & 0 & $-\frac{1}{2}$ & $\frac{1}{2}$ & $-\frac{1}{2}$ & 0 & $(1,1)$ & $(1)$ & 0 & $\frac{1}{2}$ & 0 & $-\frac{1}{2}$ \\
$V_{49}$ & $(1,1)$ & 0 & 1 & 0 & 0 & $-\frac{1}{2}$ & $\frac{1}{2}$ & $\frac{1}{2}$ & 0 & $(1,1)$ & $(1)$ & 0 & $\frac{1}{2}$ & 0 & $-\frac{1}{2}$ \\
$V_{50}$ & $(1,1)$ & 0 & -1 & 0 & 0 & $\frac{1}{2}$ & $\frac{1}{2}$ & $-\frac{1}{2}$ & 0 & $(1,1)$ & $(1)$ & 0 & $-\frac{1}{2}$ & 0 & $\frac{1}{2}$ \\
$V_{51}$ & $(1,2)$ & 0 & 0 & 0 & 0 & $-\frac{1}{2}$ & $-\frac{1}{2}$ & $\frac{1}{2}$ & 0 & $(1,1)$ & $(1)$ & 0 & $-\frac{1}{2}$ & 0 & $\frac{1}{2}$ \\
$V_{52}$ & $(1,2)$ & 0 & 0 & 0 & 0 & $\frac{1}{2}$ & $-\frac{1}{2}$ & $-\frac{1}{2}$ & 0 & $(1,1)$ & $(1)$ & 0 & $\frac{1}{2}$ & 0 & $-\frac{1}{2}$ \\
\hline \hline
\end{tabular}

Table I.a Fractionally charged states of class A with electric charges $\pm \frac{1}{2} .(C$ and $L$ in column three denote the observable sector $S U(3)_{C}$ and $S U(2)_{L}$.)

\begin{tabular}{|c|rrrrrrrrr|rrrrrr|}
\hline \hline State & $(C, L)$ & $Q_{C}$ & $Q_{L}$ & $Q_{1}$ & $Q_{2}$ & $Q_{3}$ & $Q_{4}$ & $Q_{5}$ & $Q_{6}$ & $S O(4)$ & $S U(3)$ & $Q_{H}$ & $Q_{7}$ & $Q_{8}$ & $Q_{9}$ \\
\hline$H_{1}$ & $(1,1)$ & $\frac{3}{4}$ & $\frac{1}{2}$ & $\frac{1}{4}$ & $\frac{1}{4}$ & $\frac{1}{4}$ & $-\frac{1}{2}$ & $\frac{1}{2}$ & 0 & $(2,1)$ & $(1)$ & $\frac{3}{4}$ & $\frac{1}{4}$ & 0 & 0 \\
$H_{2}$ & $(1,1)$ & $-\frac{3}{4}$ & $-\frac{1}{2}$ & $-\frac{1}{4}$ & $-\frac{1}{4}$ & $-\frac{1}{4}$ & $-\frac{1}{2}$ & $-\frac{1}{2}$ & 0 & $(2,1)$ & $(1)$ & $-\frac{3}{4}$ & $-\frac{1}{4}$ & 0 & 0 \\
$H_{3}$ & $(1,1)$ & $\frac{3}{4}$ & $\frac{1}{2}$ & $\frac{1}{4}$ & $\frac{1}{4}$ & $\frac{1}{4}$ & $\frac{1}{2}$ & $\frac{1}{2}$ & 0 & $(1,1)$ & $(1)$ & $-\frac{3}{4}$ & $-\frac{1}{4}$ & $-\frac{1}{2}$ & $\frac{1}{2}$ \\
$H_{4}$ & $(1,1)$ & $-\frac{3}{4}$ & $-\frac{1}{2}$ & $-\frac{1}{4}$ & $-\frac{1}{4}$ & $-\frac{1}{4}$ & $\frac{1}{2}$ & $-\frac{1}{2}$ & 0 & $(1,1)$ & $(1)$ & $\frac{3}{4}$ & $\frac{1}{4}$ & $\frac{1}{2}$ & $-\frac{1}{2}$ \\
$H_{5}$ & $(1,1)$ & $\frac{3}{4}$ & $\frac{1}{2}$ & $\frac{1}{4}$ & $\frac{1}{4}$ & $\frac{1}{4}$ & $\frac{1}{2}$ & $-\frac{1}{2}$ & 0 & $(1,1)$ & $(1)$ & $-\frac{3}{4}$ & $-\frac{1}{4}$ & $\frac{1}{2}$ & $-\frac{1}{2}$ \\
$H_{6}$ & $(1,1)$ & $-\frac{3}{4}$ & $-\frac{1}{2}$ & $-\frac{1}{4}$ & $-\frac{1}{4}$ & $-\frac{1}{4}$ & $\frac{1}{2}$ & $\frac{1}{2}$ & 0 & $(1,1)$ & $(1)$ & $\frac{3}{4}$ & $\frac{1}{4}$ & $-\frac{1}{2}$ & $\frac{1}{2}$ \\
$H_{7}$ & $(1,1)$ & $\frac{3}{4}$ & $\frac{1}{2}$ & $\frac{1}{4}$ & $-\frac{1}{4}$ & $-\frac{1}{4}$ & $-\frac{1}{2}$ & 0 & $\frac{1}{2}$ & $(1,1)$ & $(1)$ & $-\frac{3}{4}$ & $-\frac{1}{4}$ & $\frac{1}{2}$ & $\frac{1}{2}$ \\
$H_{8}$ & $(1,1)$ & $-\frac{3}{4}$ & $-\frac{1}{2}$ & $-\frac{1}{4}$ & $\frac{1}{4}$ & $\frac{1}{4}$ & $-\frac{1}{2}$ & 0 & $-\frac{1}{2}$ & $(1,1)$ & $(1)$ & $\frac{3}{4}$ & $\frac{1}{4}$ & $-\frac{1}{2}$ & $-\frac{1}{2}$ \\
$H_{9}$ & $(1,1)$ & $\frac{3}{4}$ & $\frac{1}{2}$ & $\frac{1}{4}$ & $-\frac{1}{4}$ & $-\frac{1}{4}$ & $-\frac{1}{2}$ & 0 & $-\frac{1}{2}$ & $(1,1)$ & $(1)$ & $-\frac{3}{4}$ & $-\frac{1}{4}$ & $-\frac{1}{2}$ & $-\frac{1}{2}$ \\
$H_{10}$ & $(1,1)$ & $-\frac{3}{4}$ & $-\frac{1}{2}$ & $-\frac{1}{4}$ & $\frac{1}{4}$ & $\frac{1}{4}$ & $-\frac{1}{2}$ & 0 & $\frac{1}{2}$ & $(1,1)$ & $(1)$ & $\frac{3}{4}$ & $\frac{1}{4}$ & $\frac{1}{2}$ & $\frac{1}{2}$ \\
$H_{11}$ & $(1,1)$ & $\frac{3}{4}$ & $\frac{1}{2}$ & $\frac{1}{4}$ & $-\frac{1}{4}$ & $-\frac{1}{4}$ & $\frac{1}{2}$ & 0 & $\frac{1}{2}$ & $(1,2)$ & $(1)$ & $\frac{3}{4}$ & $\frac{1}{4}$ & 0 & 0 \\
$H_{13}$ & $(1,1)$ & $-\frac{3}{4}$ & $-\frac{1}{2}$ & $-\frac{1}{4}$ & $\frac{1}{4}$ & $\frac{1}{4}$ & $\frac{1}{2}$ & 0 & $-\frac{1}{2}$ & $(1,2)$ & $(1)$ & $-\frac{3}{4}$ & $-\frac{1}{4}$ & 0 & 0 \\
\hline \hline
\end{tabular}

Table I.b Fractionally charged states of class B with electric charges $\pm \frac{1}{2}$. 


\begin{tabular}{|c|rrrrrrrrr|rr|rrrr|}
\hline \hline State & $(C, L)$ & $Q_{C}$ & $Q_{L}$ & $Q_{1}$ & $Q_{2}$ & $Q_{3}$ & $Q_{4}$ & $Q_{5}$ & $Q_{6}$ & $S O(4)$ & $S U(3)$ & $Q_{H}$ & $Q_{7}$ & $Q_{8}$ & $Q_{9}$ \\
\hline$H_{33}$ & $(3,1)$ & $-\frac{1}{4}$ & $-\frac{1}{2}$ & $-\frac{1}{4}$ & $-\frac{1}{4}$ & $-\frac{1}{4}$ & 0 & 0 & $-\frac{1}{2}$ & $(1,1)$ & $(1)$ & $\frac{3}{4}$ & $-\frac{1}{4}$ & $\frac{1}{2}$ & 0 \\
$H_{40}$ & $(\overline{3}, 1)$ & $\frac{1}{4}$ & $\frac{1}{2}$ & $\frac{1}{4}$ & $-\frac{1}{4}$ & $-\frac{1}{4}$ & 0 & $-\frac{1}{2}$ & 0 & $(1,1)$ & $(1)$ & $-\frac{3}{4}$ & $\frac{1}{4}$ & $\frac{1}{2}$ & 0 \\
\hline \hline
\end{tabular}

Table I.c Fractionally charged states of class $\mathrm{C}$ with electric charges $\pm \frac{1}{3}$.

\begin{tabular}{|l|l|}
\hline \hline Sector & States \\
\hline $\mathbf{1}+\mathbf{b}_{1}+\beta+2 \gamma$ & $V_{41}$ to $V_{46}$ \\
$\mathbf{I}+\beta+2 \gamma$ & $V_{47}$ to $V_{52}$ \\
$\pm \gamma$ & $H_{1}$ to $H_{2}$ \\
$\mathbf{I} \pm \gamma$ & $H_{3}$ to $H_{6}$ \\
$\mathbf{1}+\mathbf{b}_{4} \pm \gamma$ & $H_{7}$ to $H_{10}$ \\
$\mathbf{I}+\mathbf{1}+\mathbf{b}_{4} \pm \gamma$ & $H_{11}$ to $H_{13}$ \\
$\mathbf{b}_{3}+\beta \pm \gamma$ & $H_{33}$ \\
$\mathbf{b}_{1}+\mathbf{b}_{2}+\mathbf{b}_{4}+\beta$ & $H_{40}$ \\
\hline \hline
\end{tabular}

Table I.d Fractionally charged state sectors. 


\section{References}

[1] E. Witten, Nucl. Phys. B471 (1996) 135;

D.V. Nanopoulos, "M-Phenomenology," CTP-TAMU-42/97, ACT-15/97, hepth/9711080].

[2] A.E. Faraggi, D.V. Nanopoulos, and K. Yuan, Nucl. Phys. B335 (1990) 347.

[3] A.E. Faraggi, Phys. Rev. D46 (1992) 3204.

[4] G. Cleaver, M. Cvetič, J.R. Espinosa, L. Everett, and P. Langacker, Nucl. Phys. B525 (1998) 3, hep-th/9711178]; "Flat Directions in Three Generation String Models," UPR-0784T, [hep-th/9805133].

[5] For general discussions of anomalous $U(1)$ in string models see, e.g., T. Kobayashi and H. Nakano, Nucl. Phys. B496 (1997) 103, hep-th/9612066; G.B. Cleaver, Nucl. Phys. B (Proc. Suppl.) 62A-C (1998) 161 hep-th/9708023;

G.B. Cleaver and A.E. Faraggi, UFIFT-HEP-97-28, UPR-0773-T, hepph/9711339;

A.E. Faraggi, Phys. Lett. B426 (1998) 315, hep-ph/9801409];

L.E. Ibáñez, R. Rabadan, and A.M. Uranga, hep-th/9808139;

P. Ramond, Proceedings of Orbis Scientiae '97 II, Dec. 1997, Miami Beach, thepph/9808488;

W. Pokorski and G.G. Ross, [hep-ph/9809537] and references contained within each.

[6] M. Dine, N. Seiberg and E. Witten, Nucl. Phys. B289 (1986) 585;

J. Atick, L. Dixon and A. Sen, Nucl. Phys. B292 (1987) 109.

[7] I. Antoniadis, J. Ellis, J. Hagelin and D.V. Nanopoulos Phys. Lett. B231 (1989) 65 ;

I. Antoniadis. G.K. Leontaris and J. Rizos, Phys. Lett. B245 (1990) 161;

J.L. Lopez, D.V. Nanopoulos and K. Yuan, Nucl. Phys. B399 (1993) 654;

A.E. Faraggi, Phys. Lett. B339 (1994) 223.

[8] A.E. Faraggi, Phys. Lett. B278 (1992) 131.

[9] M. Luty, Phys. Rev. D53 (1996) 3399, hhep-th/9506098];

T. Gherghetta, C. Kolda, and S. Martin, Nucl. Phys. B468 (1996) 37, hepth/9510370];

E. Dudas, C. Grojean, S. Pokorski, and C.A. Savoy, Nucl. Phys. B481 (1996) 85, hep-ph/9606383;

P. Binetruy, N. Irges, S. Lavignac, P. Ramond, Phys. Lett. B403 (1997) 38, hep-ph/9612442];

N. Irges and S. Lavignac Phys. Lett. B424 (1998) 293, hep-ph/9712239. 
[10] G. Cleaver, "Mass Hierarchy and Flat Directions in String Models," UPR-0795T. Proceedings of Orbis Scientiae '97 II, Dec. 1997, Miami Beach.

[11] I. Antoniadis, C. Bachas, and C. Kounnas, Nucl. Phys. B289 (1987) 87;

H. Kawai, D.C. Lewellen, and S.H.-H. Tye, Nucl. Phys. B288 (1987) 1.

[12] A.E. Faraggi and D.V. Nanopoulos, Phys. Rev. D48 (1993) 3288.

[13] A.E. Faraggi, Phys. Lett. B274 (1992) 47.

[14] A.E. Faraggi, Nucl. Phys. B387 (1992) 239; hep-th/9708112.

[15] S.M. Barr, Phys. Lett. B112 (1982) 219;

J.P. Derendinger, J.E. Kim and D.V. Nanopoulos, Phys. Lett. B139 (1984) 170;

I. Antoniadis, J. Ellis, J.S. Hagelin and D.V. Nanopoulos, Phys. Lett. B194 (1987) 231.

[16] J. March-Russell, "The Fayet-Iliopoulos term in Type-I string theory and Mtheory," IASSNS-HEP-97/128, CERN-TH/98-198, hhep-ph/9806425.

[17] G. Cleaver, A.E. Faraggi and D.V. Nanopoulos, papers in preparation.

[18] S. Kalara, J. López, and D.V. Nanopoulos, Phys. Lett. B245 (1990) 421; Nucl. Phys. B353 (1991) 650;

A.E. Faraggi, Nucl. Phys. B487 (1997) 55.

[19] D. Bailin, D. Dunbar, and A. Love, Phys. Lett. B219 (1989) 76;

J. Rizos and K. Tamvakis, Phys. Lett. B262 (1991) 227.

[20] G. Cleaver, M. Cvetič, J.R. Espinosa, L. Everett, and P. Langacker, Phys. Rev. D57 (1998) 2701, hep-ph/9705391.

[21] M. Cvetič, L. Everett, J. Wang, "Units and Numerical Values of the Effective Couplings in Perturbative Heterotic String Theory," UPR-0810-T, hepph/9808321].

[22] G. Cleaver, M. Cvetič, J.R. Espinosa, L. Everett, P. Langacker and J. Wang, "Physics Implications of Flat Directions in Free Fermionic Superstring Models I: Mass Spectrum and Couplings," CERN-TH/98-243, UPR-0811-T, IEM-FT177/98, UM-TH-98/17, hep-ph/9807479; "Physics Implications of Flat Directions in Free Fermionic Superstring Models II: Renormalization Group Analysis," UPR-0814-T, UM-TH-98/18, hep-ph/9811355. 